REVISTA PROYECCIONES: 179-200.

JORNADAS MATEMATICAS, AGOSTO 1983.

\title{
ESPACIOS UNIFORMES
}

\section{Dr. SAMUEL NAVARRO HERNANDEZ *}

NOCIONES BASICAS SOBRE FILTROS.

Sea $x$ un conjunto no vacío, $F \subseteq P(x)$ es un filtro sobre $x$ si
1) Dados $A, B$ en $F$ se tiene $A \cap B \in F$
2) Si $A \in F$ y $A \subset B$ entonces $B \in F$
3) $\phi \notin F$

Un conjunto $B \subseteq P(x), B \neq \phi$ es una base de filtro sobre $\mathrm{x}$ si

1) Dados $A, B$ en $B$ se tiene $A \cap B \in B$

2) $\phi \not B B$

Un conjunto $S \underline{c} P \quad(\mathrm{x}), S \neq \phi$ es una subbase de filtro sobre $\mathrm{x}$ si

* Profesor Departamento de Matematica y Ciencia de la Computación, Facultad de Ciencia. Universidad de Santiago de Chile. 
1) Toda familia finita en $S$ tiene intersección no vacía.

CONSTRUCCION DE FUUTROS Y BASES DE FHLTRO.

A) Si $B$ es base de filtro entonces

$$
F=\{A \underline{c} X / A \underline{\simeq} B \text { para algún } B \text { en } B\}
$$

es un filtro que se llama filtro generado por $B$.

Si $S$ es subbase de filtro entonces

$$
B=\left\{B \underline{c} \times / B=\bigcap_{i=j}^{n} s_{i}, s_{i} \varepsilon S\right\}
$$

es una base de filtro.

Obs.: Un filtro puede tener más de una base. En un conjunto $\mathrm{X}$ puede haber tantos filtros como permita la cardinalidad de $\mathrm{X}$.

Notación. $\Phi(\mathrm{X})=\{F / F$ es un filtro sobre $\mathrm{x}\}(\Phi(\mathrm{X}), \underline{\mathrm{c}})$ es ordenado.

Ejemplos:

1) Dado $\mathrm{x} \in \mathrm{X},\{\mathrm{x}\}$ es una base de filtro.

Se usa la notación $\dot{x}$ para el filtro generado por $\{\mathrm{x}\}$.

2) Si $x$ es infinito

$B=\left\{A / A^{C}\right.$ finito $\}$ es base de filtro.

3) Si $\mathrm{x}$ es espacio topolбgico y $\mathrm{x} \varepsilon \mathrm{x}$

$\mathrm{V}(\mathrm{X})=\{$ las vecindades abiertas de $\mathrm{x}\}$ es una base de filtro. 
4) Si $\left\{x_{n}\right\}$ es una sucesión en $x\left\{_{n}\right\}_{n \in \mathbb{N}}$ donde $B_{n}=\left\{x_{m} / m \geqslant n\right\}$, es una base de filtro.

5) $F=\{\mathrm{X}\}$ es un filtro.

6) $\operatorname{Si} \mathrm{x}=\mathbb{N}$ $F=\left\{A_{n} / A_{n}=\{m / m \geqslant n\}\right\}$ se llama filtro de Frèchet.

7) Si $\mathrm{x}$ es preordenado filtrante según $\sigma$ y a $\varepsilon \mathrm{x}$ anotamos el conjunto $S(a)=\{x \in X / a \sigma x\}$ que llamamos sección de a. $B=\{\mathrm{S}(\mathrm{a}) / \mathrm{a} \varepsilon \mathrm{X}\}$ es una base de filtro, el filtro generado se llama filtro de las secciones de $\mathrm{X}$.

Def.: Un ultrafiltro es un elemento maximal de $(\Phi(x), \underline{c})$

Obs.: Para cada filtro hay un ultrafiltro que lo contiene (Zorn). Una forma de caracterizar los ultrafiltros es:

1) $F$ es un ultrafiltro ssi $A \cup B \in F$ $\Longrightarrow A \in F$ б $\quad$ B $\in F$

2) $F$ es un ultrafiltro ssi

A $\cap F \neq \phi \forall F \varepsilon F$ implica $A \varepsilon F$ 


\section{FILTROS INDUCIDOS.}

Sea $\mathrm{X}$ un conjunto, $\mathrm{S} \underline{\mathrm{c}} \mathrm{X}, \mathrm{F}$ un filtro sobre $\mathrm{x}$, definimos el conjunto

$$
F_{\mathrm{S}}=\{\mathrm{A} \cap \mathrm{S} / \mathrm{A} \in \mathrm{F}\}
$$

que llamamos traza de $F$ sobre $\mathrm{S}$.

Obs.:

$$
\begin{aligned}
& F_{S} \text { es un filtro en S ssi } \\
& F \text { está frecuentemente en } S \text { (i - e A } \cap S \neq \phi \forall A \in F \text { ) }
\end{aligned}
$$

Producto:

Si $\mathrm{x}, \mathrm{X}^{\prime}$ son dos conjuntos

$B, B^{\prime}$ dos bases de filtro en $\mathrm{X} y \mathrm{X}^{\prime}$ respectivamente.

$$
B=\left\{B_{1} \times B_{2} / B_{1} \in B, B_{2} \in B^{\prime}\right\}
$$

es una base de filtro en $\mathrm{x} \times \mathrm{X}^{\prime}$.

(El caso infinito también vale)

$$
B=\left\{\mathbb{I B}_{i} / B_{i} \varepsilon B, B_{i} \neq x \text { sólo en un número finito de Índices }\right\}
$$

es una base de filtro en $\mathbb{I x}_{i}$

Dados $\mathrm{X}, \mathrm{X}^{\prime}$ conjuntos

$f: x \longrightarrow x^{\prime}$ una función.

y $F$ un filtro en $\mathrm{x}$ siempre se tiene que

$f(F)$ es base de filtro en $X^{\prime}$. 


\section{ESPACIOS UNIFORMES.}

Sea $\mathrm{x}$ conjunto no vacío. Para $\mathrm{W}, \mathrm{V} \mathrm{c} \mathrm{x} \times \mathrm{x}$ definimos:

$$
\begin{aligned}
& \mathrm{W}^{-1}=\{(\mathrm{y}, \mathrm{x}) \varepsilon \mathrm{x} \times \mathrm{x} /(\mathrm{x}, \mathrm{y}) \varepsilon \mathrm{W}\} \\
& \mathrm{V}^{\circ} \mathrm{W}=\left\{(\mathrm{x}, \mathrm{z}) \varepsilon \mathrm{x} \mathrm{x} / \mathrm{B}^{\mathrm{y}} \varepsilon \mathrm{x} \partial(\mathrm{x}, \mathrm{y}) \varepsilon \mathrm{W} \wedge(\mathrm{y}, \mathrm{z}) \varepsilon \mathrm{v}\right\}
\end{aligned}
$$

La diagonal de $\mathrm{x} \times \mathrm{x}$ es:

$$
\Delta=\{(\mathrm{x}, \mathrm{x}) / \mathrm{x} \varepsilon \mathrm{x}\}
$$

Sea $U$ un filtro sobre $\mathrm{X} \times \mathrm{x}$ satisfaciendo los axiomas:

1). $\forall \mathrm{W} \in U, \Delta \mathrm{CW}$

2) $W \in U \Longrightarrow W^{-1} \in U$

3) $\forall \mathrm{W} \varepsilon U, \exists \mathrm{V} \in U$ ЭV $\circ \mathrm{V} \subset \mathrm{W}$

Decimos que el filtro $U$ define una uniformidad o una estructura uniforme sobre $\mathrm{X}$ y cada $W \in U$ es llamada una vecindad de la uniformidad. (conector).

Def.:

El par (X,U) se llama Espacio Uniforme.

Def.:

Una estructura uniforme $U$ sobre $X$ es Hausdorff si

$\Omega\{W / W \in U\}=\Delta$

Ejemplo:

1) $U=\{\mathrm{A} \times \mathrm{B} \leq \mathrm{C} \times \mathrm{x} / \mathrm{A} \times \mathrm{B} \leq \Delta\}$ 
2) $U=\{x \times x\}$

3) Sea $\mathrm{x}=\mathbb{R}^{+}$y sea $H=\left\{\mathrm{v} \alpha \in \mathbb{R}^{+} \mathrm{x} \mathbb{R}^{+} / \alpha \in \mathbb{R}^{+}\right\}$donde $\mathrm{v}_{\alpha}=\left\{(\mathrm{x}, \mathrm{y}) \in \mathbb{R}^{+} \mathrm{x} \mathbb{R}^{+} /|\mathrm{x}-\mathrm{y}|<\alpha\right\}$

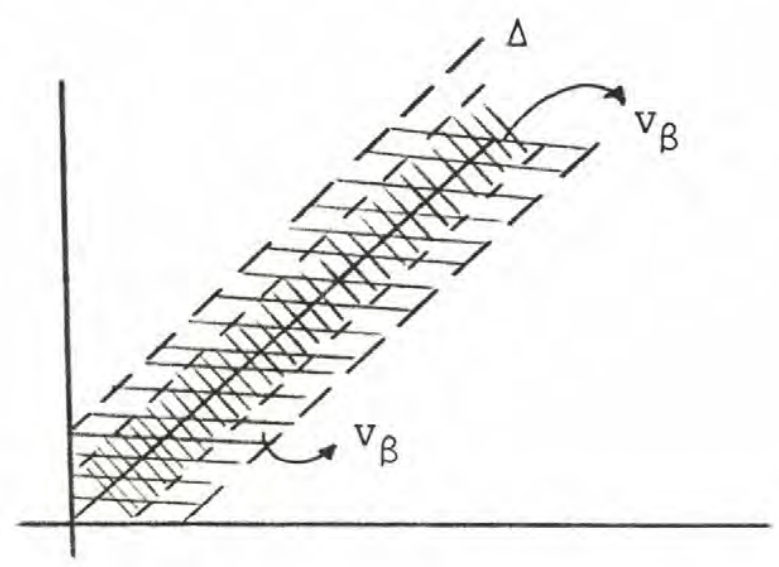

Entonces $H$ es una base de filtro sobre $\mathrm{x} \times \mathrm{x}$ y el filtro $U$ generado por $H$ define una uniformidad sobre $X=\mathbb{R}^{+}$.

Dados $X$ conjunto, $U$ estructura uniforme en $\mathrm{x} \times \mathrm{x}$ se define $V$ como la familia de subconjuntos $G$ c $X$ tal que: si $X \in G$ entonces $\exists \mathrm{W} \varepsilon U \exists\{\mathrm{y} \varepsilon X /(\mathrm{x}, \mathrm{y}) \varepsilon \mathrm{W}\}$ c $G$

Prop. :

Sean $G_{i} \in V, i=1, \ldots, \mathrm{n}$ entonces

$\prod_{i=1}^{n} \quad G_{i} \in V$

$$
\text { Sea: } X \quad \varepsilon \prod_{i=1}^{n} G_{i} \Longrightarrow X \varepsilon G_{i} \quad \forall i=1, \ldots, n \Longrightarrow \exists \mathrm{w}_{i} \varepsilon U
$$

tal que: $\left\{y \in \mathrm{x} /(\mathrm{x}, \mathrm{y}) \in \mathrm{w}_{\mathrm{i}}\right\} \quad \mathrm{c} \quad G_{\mathrm{i}} \quad \forall i=1, \ldots, \mathrm{n} \Longrightarrow$

$\sum_{i=1}^{n}\left\{y \in x /(x, y) \in w_{i}\right\} \quad c \quad n_{i=1}^{n} \quad G_{i}$ 


$$
\begin{aligned}
& \text { Como } U \text { es filtro, } \mathrm{n}_{i=1}^{\mathrm{n}} \mathrm{w}_{i}=\widetilde{\mathrm{W}} \varepsilon U \Longrightarrow\{\mathrm{y} \varepsilon X /(\mathrm{x}, \mathrm{y}) \varepsilon \tilde{\mathrm{W}}\} \\
& \mathrm{n} \quad \mathrm{n} \quad \mathrm{n} \\
& \text { c } \bigcap_{i=1}\left\{y \in X /(x, y) \in w_{i}\right\} \quad c \quad \bigcap_{i=1}^{n} G_{i} \Longrightarrow \underset{i=1}{\cap} G_{i} \varepsilon V
\end{aligned}
$$

Prop. :

Dado $G_{i} \in V$, $i \varepsilon I$, conjunto de índices arbitrario

$\Longrightarrow \underset{i \varepsilon I}{U} G_{i} \in V$

$$
\begin{aligned}
& \text { Sea } X \in \underset{i \in I}{U} G_{i} \Longrightarrow \exists j \varepsilon I \text { tal que: } X \varepsilon G_{j} \Longrightarrow \exists \tilde{W} \varepsilon U 3 \\
& \{y \in X /(x, y) \varepsilon \tilde{W}\} \quad c_{j} \Longrightarrow\left\{y \in X /(x, y) \varepsilon \tilde{W} \subset \underset{i \in I}{\sim} \mathrm{C}_{i} \Longrightarrow\right. \\
& \underset{i \varepsilon I}{U} G_{i} \in V
\end{aligned}
$$

Además $X \in V$ puesto que $\forall w \in U \forall \mathrm{x} \varepsilon \mathrm{x}\{\mathrm{y} \varepsilon X /(\mathrm{x}, \mathrm{y}) \varepsilon \mathrm{w}\}$ c $X \Longrightarrow X \in V$

También $\phi \varepsilon V$, pues sino:" $\exists \times \varepsilon \phi$ tal que $\forall W \in B$

$\{y \in X /(x, y) \in W\} \not \phi^{\prime \prime}(\Longrightarrow$

Obs.:

$V$ define una topología $T_{v}$ sobre $X 3 \forall \times \varepsilon X$ la familia $\mathrm{W}(\mathrm{x})=\{\mathrm{y} \varepsilon X /(\mathrm{x}, \mathrm{y}) \varepsilon \mathrm{W}\}$ donde $\mathrm{W}$ recorre $U$ es una base de vecindades de $X$. 

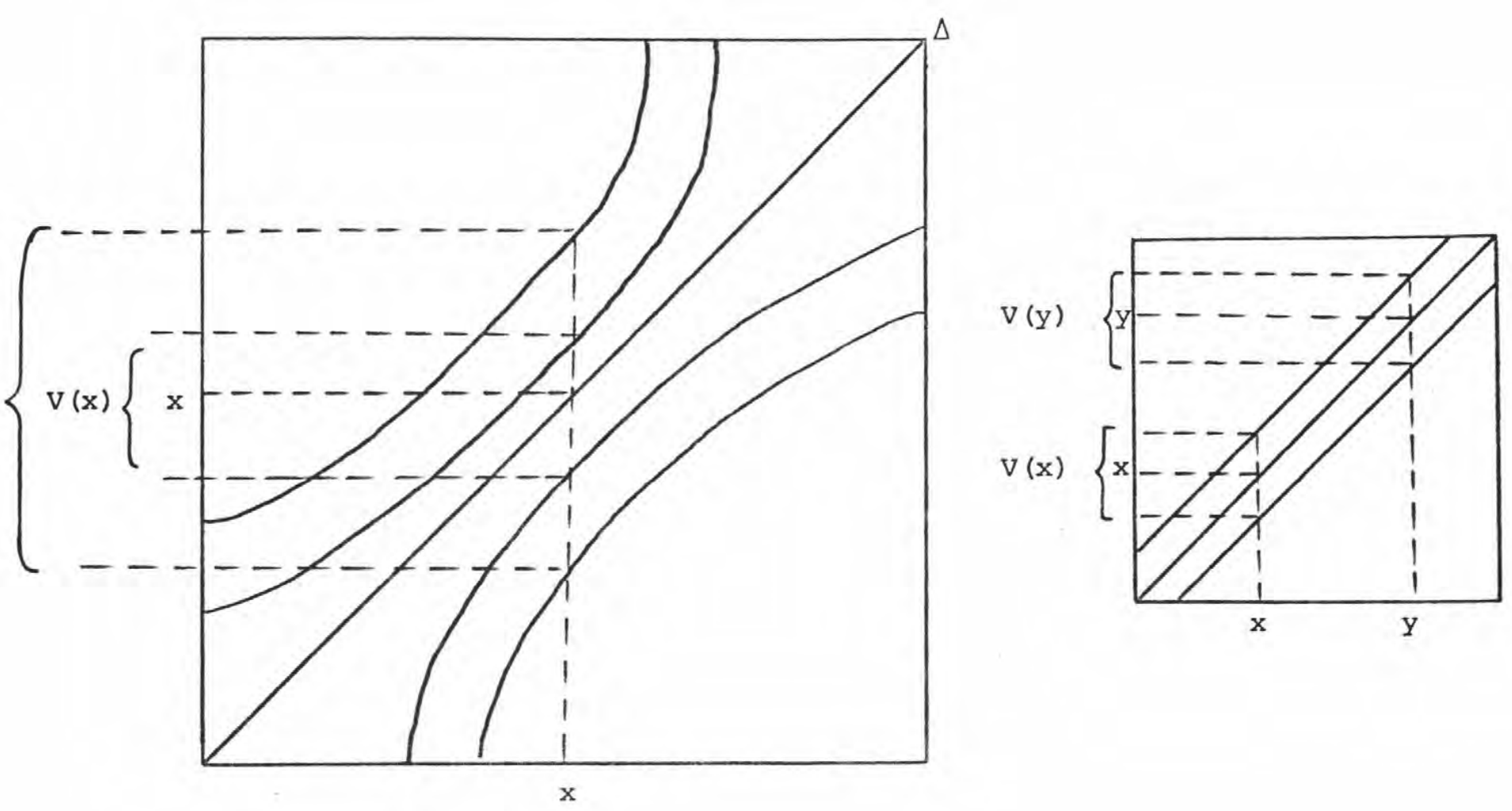

Def.:

a) Un espacio topológico $X$ es uniformizable si su topología puede ser derivada de una uniformidad sobre $X$.

b) La topología derivada de la uniformidad es una topología de Hausdorff si la uniformidad es Hausdorff.

Prop.:

Un espacio topológico de Hausdorff es uniformizable ssi es completamente regular ( $X$ es completamente regular si F c X cerrado y $p \varepsilon X \backslash F, \exists f: X \longrightarrow[0,1]$ continua t.q. $f(p)=0 y f(F)=1)$

Definición.

Sean $\left(X, U_{1}\right)\left(Y, U_{2}\right)$ espacios uniformes. Una aplicación 
$\mathrm{f}: X \rightarrow \mathrm{Y}$ es uniformemente continua si $\forall \mathrm{V}$ en $U_{1} \equiv \mathrm{U}$ conector en $U_{1}$ tal que $(x, y) \in U \Longrightarrow(f(x), f(y)) \varepsilon V$

Prop.:

Cada aplicación uniformemente continua es continua.

Dem.: Trivial.

Obs.:

1) Los espacios uniformes $X, Y$ son isomorfos si $\exists \mathrm{f}: \mathrm{X} \longrightarrow \mathrm{Y}$ biyec tiva tal que $f \mathrm{y}^{-1}$ son uniformemente continuas.

f es llamado "isomorfismo uniforme".

2) Si $U_{1}$ y $U_{2}$ son dos filtros sobre $\mathrm{x} x \mathrm{x}$ que definen uniformidades so bre $X$ y si $U_{1}$ c $U_{2}$ decimos que la uniformidad definida por $U_{1}$ menos fina que la definida por $U_{2}$.

3) Si $X$ es un conjunto $\left\{X_{\alpha} / \alpha \varepsilon A\right\}$ familia de espacios uniformes $y$ $f_{\alpha}: X \longrightarrow X_{\alpha^{\prime}}$ entonces, existe una uniformidad menos fina sobre $X$ para la cual $f_{\alpha}, \alpha \in A$ son uniformemente continuas.

Como caso particular la uniformidad producto sobre $X=\underset{\alpha \varepsilon A}{I} X_{\alpha}$

Similarmente si $X$ es un espacio uniforme y S c $X$ la unifor midad inducida es la uniformidad sobre $S$ menos fina para la cual la in yección canónica $i: s \longrightarrow X$ es uniformemente continua.

Def.:

(X, UL esp. uniforme, $F$ un filtro sobre $\mathrm{X}$ es convergente a $x \in \mathrm{X}$, si $F$ es más fino que $\{\mathrm{W}(\mathrm{x}) / \mathrm{W} \in U\}$

Def.:

Sea $X$ espacio uniforme. Un filtro $F$ sobre $X$ es un filtro de Cauchy si para cada vecindad $\mathrm{V} \in U \exists \mathrm{U} \in F$ t.q. $F \times F$ c . 
Si cada filtro de Cauchy converge (a un elemento de $X$ ) entonces $\mathrm{x}$ es llamado completo.

Obs.:

1) Si $f:\left(X, U_{1}\right) \longrightarrow\left(Y, U_{2}\right)$ es uniformemente continua y $F$ filtro de Cauchy en $X \Longrightarrow \mathrm{f}(F)$ filtro de Cauchy en $\mathrm{Y}$.

2) Para cada espacio uniforme $X$ podemos construir un espacio uniforme completo $\tilde{X}$ tal que $X$ es uniformemente isomorfo a un subespacio denso de $\tilde{X}$ y tal que $\tilde{X}$ es Hausdorff si $X$ lo es. SiX es Hausdorff, entonces $\tilde{X}$ es determinado por estas propiedades por el isomorfismo y es llamado la completación de $X$.

Una base de vecindades de uniformidad de $\vec{X}$ puede ser obte nida tomando las clausuras (en la topología producto $\tilde{X} \times \vec{X}$ ) de la base de vecindades de uniformidad de $X$.

3) Si cada sucesión de Cauchy en $X$ converge, entonces $X$ es llamado semicompleto o secuencialmente completo.

4) Si $X$ es un espacio uniforme completo y A un subespacio cerrado en tonces el espacio uniforme $A$ es completo.

5) Si $X$ es un espacio uniforme completo y A un subespacio completo en tonces $\mathrm{A}$ es cerrado en $X$.

6) Un producto de espacios uniformes es completo ssi cada espacio fac tor es completo.

7) Si $X$ es un espacio uniforme, $Y$ un espacio completo Hausdorff, $x_{0} c x y f: x_{0} \longrightarrow y$ uniformemente continua, entonces $f$ tiene una única extensión uniformemente continua: $\overline{\mathrm{f}}: \overline{\mathrm{X}_{\mathrm{O}}} \longrightarrow \mathrm{Y}$. 
EXTENSION POR CONTINUIDAD.

Teorema: Sea $X$ espacio topológico A c $X$ denso, $\mathrm{f}: \mathrm{A} \longrightarrow \mathrm{Y}$, Y regular (es decir F C Y cerrado, $\mathrm{p} \varepsilon \mathrm{Y} \backslash \mathrm{F}, \exists \mathrm{V}, \mathrm{W}$ abiertos disjun tos $\ni F \subset V y p \in W$ ) entonces $\mathrm{f}$ se extiende continuamente a $\bar{f}: X \longrightarrow Y$ ssi $\forall x \in X$,

$$
\begin{aligned}
& \lim f(Y)=Z \& Y \\
& y \in A
\end{aligned}
$$

Dem.:

$$
\begin{aligned}
& \overline{\mathrm{f}} \text { continua sobre } X \Longrightarrow \forall \mathrm{x} \varepsilon \mathrm{X} \\
& \bar{f}(x)=\lim \bar{f}(y)=\lim f(y) \\
& \mathrm{y} \longrightarrow \mathrm{x} \quad \mathrm{y} \longrightarrow \mathrm{x} \\
& y \in A \quad y \in A
\end{aligned}
$$

Recíprocamente: sea

$$
\begin{aligned}
\mathrm{f}(\mathrm{x})= & \lim \mathrm{f}(\mathrm{y}) \quad \forall \mathrm{x} \in X \\
& \mathrm{y} \longrightarrow \mathrm{x} \\
& \mathrm{x} \in \mathrm{A}
\end{aligned}
$$

$\overline{\mathrm{f}}$ está definida pues $\mathrm{Y}$ es Hausdorff Pd. $\overline{\mathrm{f}}$ es continua $\forall x \in X$

Sea $V^{\prime}$ vecindad cerrada de $\overline{\mathrm{f}}(\mathrm{x})$ en $\mathrm{Y} \Longrightarrow \exists \mathrm{V}$ vecindad abier-

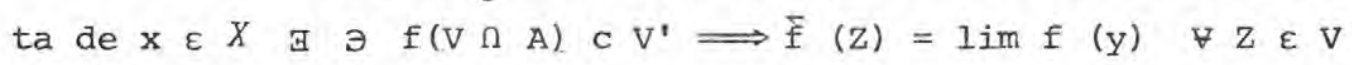

$$
\begin{aligned}
& y \longrightarrow z \\
& y \in A
\end{aligned}
$$

$\Longrightarrow \bar{f}(z) \in \overline{f(V \cap A)} \subset V^{\prime}$ puesto que $V^{\prime}$ es cerrado $y$ el resultado sigue del hecho de que vecindades cerradas de $f(x)$ forman un sistema fun damental de vecindades de $f(X)$ en $Y$. 
Prop. :

Sea $X$ espacio topológico $A \subset X$ denso $y \mathrm{f}: \mathrm{A} \longrightarrow X^{\prime}$ donde $X^{\prime}$ es esp. unif. completo $T_{2}$, entonces $f$ puede ser extendida a $X$ por continuidad ssi $\forall \times \in \in X$, la imagen bajo $f$ de la traza sobre $A$ del fil tro de vecindades de $x \in X$, es una base de filtro de cauchy en $X^{\prime}$.

Dem.:

Sigue del teorema anterior porque $X^{\prime}$ es regular y porque so bre $X^{\prime}$ los filtros de Cauchy son convergentes.

\section{LA COMPLETACION DE UN ESPACIO UNIFORME.}

Teorema:

Sea $X$ espacio uniforme. Entonces existe un espacio uniforme $\mathrm{T}_{2}$ completo $\hat{\mathrm{x}} \mathrm{y}$ una aplicación uniformemente continua $i: x \rightarrow \hat{x} t$. $q$ $(P)$ : Dada cualquier aplicación uniformemente continua $\mathrm{f}: \mathrm{X} \longrightarrow \mathrm{Y}, \mathrm{Y}$ espacio uniforme $\mathrm{T}_{2}, \exists$ ! aplicación uniformemente continua.

$$
g: \hat{x} \rightarrow Y \text { t.g } \quad f=g \circ i
$$

(e.d. i es una aplicación universal).

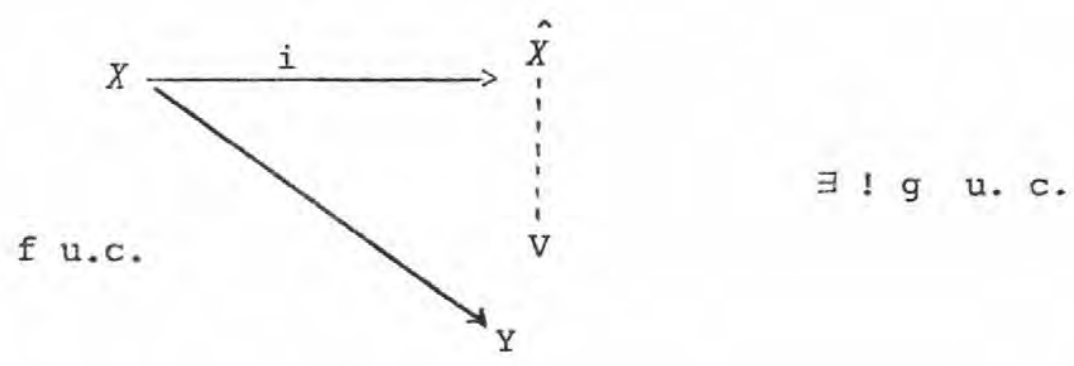

Si $\left(i_{1}, X_{1}\right)$ es otro par donde $X_{1}$ es un e.u.c. $\mathrm{T}_{2}$ e $i_{1}: X \longrightarrow x_{1}$ u.c. t.q. verifica $(P)$ entonces $\exists ! r: \hat{x} \longrightarrow x_{1}$ isomorfismo t.q $i_{1}=\gamma \circ i$ 


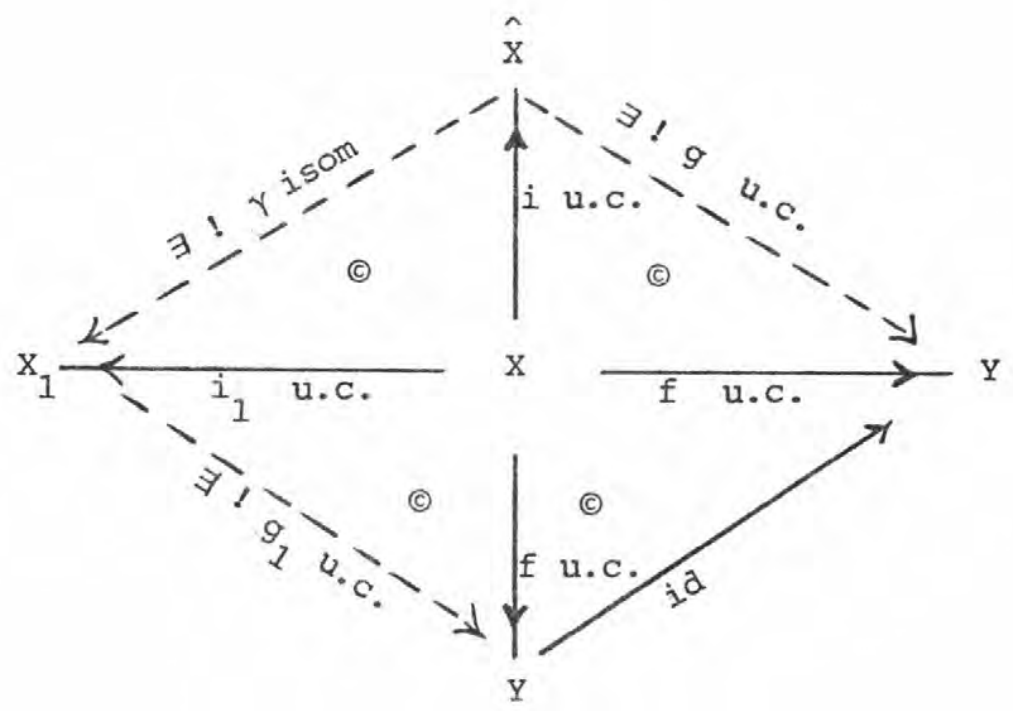

Definición:

El e.u.c. $T_{2}, \hat{X}$ del Teorema es llamađo la completación de Haus dorff de $X$, y la aplicación $i: X \longrightarrow \hat{X}$ es llamada la aplicación canó nica de $X$ en su completación de Hausdorff.

\section{Propiedades:}

1) Si $X$ es un e.u. $\mathrm{T}_{2}$ entonces $i: X \rightarrow \hat{X}$ es un isomorfismo de sobre un subespacio denso de $\hat{X}$ (esto caracteriza la completación de un espacio $\mathrm{T}_{2}$ ).

2) Si $X$ no es $T_{2}$, entonces sólo podemos asegurar que i (X) es un subespacio denso de $\hat{X}$. 
UNIFORMIDAD Y ESPACIOS VECTORIAIES TOPOLOGICOS.

Definición.

Una uniformidad sobre un espacio vectorial I es llamada invariante bajo traslación si tiene una base $N$ t $q(x, y) \varepsilon N$ es equivalente con $(x+z, y+z) \varepsilon N \quad \forall z \varepsilon L$ y $N \varepsilon N$

Proposición.

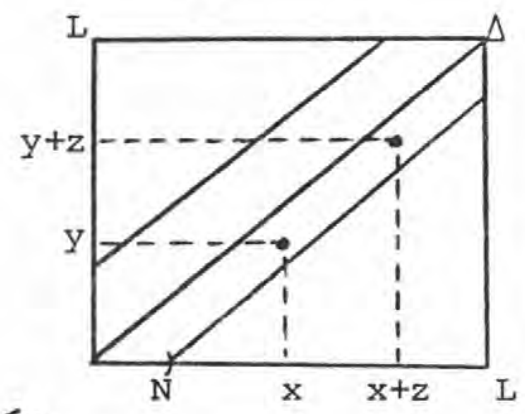

$$
(x, y) \varepsilon N \Longleftrightarrow(x+z, y+z) \varepsilon N
$$

La topología de cualquier espacio vectorial topológico puede ser derivada de una única uniformidad $N$ invariante bajo traslación. Si $B$ es cualquier base de vecindades de 0 , la familia $N_{v}=\{(x, y) / x$ - y $\varepsilon \mathrm{V}\}, \mathrm{V} \in B$ es una base para $N$.

Dem. :

Sea $(L, C)$ e.v.t. $\operatorname{con} B$ base de vecindades de 0 .

p.d. $\tilde{N}=\left\{\mathrm{N}_{\mathrm{v}} / \mathrm{v} \varepsilon B\right\}$ forman una base de filtro sobre $\mathrm{L} \times \mathrm{L}$.

i) $\hat{N} \neq \phi$ pues $\forall \mathrm{V} \varepsilon B, \circ \varepsilon \mathrm{V} \Longrightarrow(0,0) \varepsilon \mathrm{N}_{\mathrm{v}} \wedge \mathrm{N}_{\mathrm{v}} \neq \phi \quad \forall \mathrm{N}_{\mathrm{v}}$

ii) $\mathrm{N}_{\mathrm{v}}, \mathrm{N}_{\mathrm{w}} \varepsilon \tilde{N} \Longrightarrow \mathrm{V}$ c $\mathrm{W}$ ó $\mathrm{W}$ c $\mathrm{V}$ sin pérdida de generalidad

$\mathrm{V} \subset \mathrm{W} \Longrightarrow \mathrm{N}_{\mathrm{v}} \mathrm{C} \mathrm{N} \mathrm{N}_{\mathrm{v}} \cap \mathrm{N}_{\mathrm{W}}$

Luego $\tilde{I}$ es una base de filtro sobre L x L que es una base para la uniformidad $N$ invariante bajo traslación produciendo la topología $C$ de $\mathrm{L}$. 
Si $N_{1}$ es otra uniformidad con estas propiedades existe una base $M$ de $N_{1}$ consistente de conjuntos invariantes bajo traslación y $t$. q. los conjuntos

$$
\mathrm{U}_{\mathrm{m}}=\{\mathrm{x}-\mathrm{y} /(\mathrm{x}, \mathrm{y}) \in \mathrm{m}\}, \mathrm{m} \varepsilon M \text { forman una base de vecinda- }
$$
des de o para $C$, puesto que:

$$
\left(\mathrm{U}_{\mathrm{m}} \mathrm{c} \mathrm{V} \Longleftrightarrow \mathrm{mcN} \mathrm{N}_{\mathrm{v}}\right) \Longrightarrow N_{1}=N
$$

Obs.:

1) A c L es completo ssi cada filtro de Cauchy en A converge a un elemento de A (A con la uniformidad inducida).

2) Un e.v.t. L es un e.v. Hausdorff ssi I es un esp. unif. $T_{2}$.

Luego $\mathrm{I}$ es $\mathrm{T}_{2}$ ssi $\cap\{U, U \in U\}=\{0\}$, donde $U$ es cualquier base de vecindades de 0 en $L$.

3) Si L es esp. vect. top, por un subespacio de I entendemos un subes pacio $M$ (es $\operatorname{decir} M \neq \phi, M+M C M, K M \subset M$ ) provisto de la topología inducida por $L$.

$$
\text { M e.v.t es } \mathrm{T}_{2} \text { si L } 10 \text { es. }
$$

4) I esp. vect. top $\mathrm{T}_{2} \Longrightarrow$ L es completamente regular.

Teorema. (Completación de L).

Sea $\mathrm{L}$ esp. vect. top $\mathrm{T}_{2}$, entonces existe un esp. vect. top. c. $\mathrm{T}_{2} \tilde{\mathrm{L}}$ sobre $\mathrm{K}$ t.q $\mathrm{L} \mathrm{C} \tilde{\mathrm{L}}$ es denso, $\tilde{\mathrm{L}}$ es único salvo isomorfismo. Además para cualquier base $B$ de vecindades de $O$ en $L$, la familia $\tilde{B}=\{\overline{\mathrm{V}}, \mathrm{V} \in B\}$ de clausuras en $\tilde{L}$ es una base de vecindades de $\mathrm{O}$ en $\tilde{\mathrm{L}}$. 
Dem. :

Asumamos conocido la existencia de un e.u.c. $\mathrm{T}_{2} \tilde{\mathrm{L}}$ el cual contiene a L como subespacio denso, el cual es único salvo isomorfismo uni forme. Por proposición anterior:

$$
\begin{aligned}
& \begin{aligned}
+: \quad \mathrm{x} L \longrightarrow \\
(\mathrm{x}, \mathrm{y}) \longrightarrow \\
\mathrm{L}
\end{aligned} \\
& \therefore \mathrm{K} \times \mathrm{L} \longrightarrow \tilde{\mathrm{L}} \\
& (\lambda, x) \longrightarrow \lambda x \quad
\end{aligned}
$$

En efecto:

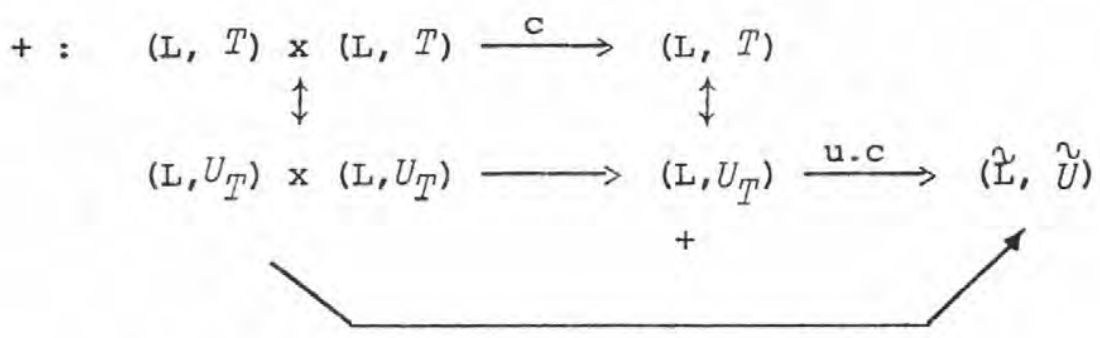

P.d. Dado $\tilde{U} \varepsilon \tilde{U}$ Ux $U \in U_{T} \times U_{T}$ t.q $U+U \subset \tilde{U}$

sea $B$ base de vecindades de $O$ en $T$, entonces

$$
\left\{\mathrm{N}_{\mathrm{v}} / \mathrm{v} \varepsilon B\right\} \text { es base de } U_{T}
$$

Por continuidad de + , dado $T \varepsilon T \exists \mathrm{S}$ x $\mathrm{S} \varepsilon T$ e $T$ t.q $\mathrm{S}+\mathrm{S}$ C T (donde $\mathrm{S}$ y $\mathrm{T}$ son vecindades de $\mathrm{O}$ ).

Pero asociado a $\mathrm{S}$ tenemos $\mathrm{N}_{\mathrm{S}} \mathrm{Y}$ a $\mathrm{T}, \mathrm{N}_{\mathrm{T}}$. Ahora, dado $\tilde{U} \varepsilon \tilde{U} \exists U \in U_{T}$ tal que U c $\tilde{U}$ pero $\left\{\mathrm{N}_{\mathrm{v}}\right\}$ es base de $U_{T}$

$$
\Longrightarrow \exists \mathrm{T} \in T \text { t.q } \quad \mathrm{N}_{\mathrm{T}} \subset \mathrm{U} \text {. }
$$

Luego, si probamos que:

$$
\mathrm{N}_{\mathrm{S}}+\mathrm{N}_{\mathrm{S}} \mathrm{c} \mathrm{N}_{\mathrm{T}} \mathrm{c} \mathrm{U} \subset \tilde{U} \text { tendríamos que dado } \tilde{U} \varepsilon \cdot \tilde{U} \text {, }
$$


$\exists \mathrm{N}_{\mathrm{S}} \times \mathrm{N}_{\mathrm{S}} \varepsilon U_{T} \times U_{T}$ t.q $\mathrm{N}_{\mathrm{S}}+\mathrm{N}_{\mathrm{S}} \mathrm{C} \tilde{U}$, lo que probaría la continuidad uni forme.

En efecto,

(a, b) $\left.\varepsilon \mathrm{N}_{\mathrm{S}} \Longrightarrow \mathrm{a}-\mathrm{b} \varepsilon \mathrm{s}\right\} \Longrightarrow$

$(c, d) \varepsilon N_{S} \Longrightarrow c-d \varepsilon s$

$(a, b)+(c, d)=(a+c, b+d) \varepsilon N_{T}$, puesto que

$a+c-(b+d)=\underbrace{(a-b)}_{\varepsilon s}+\underbrace{(c-d)}_{s, s)} \varepsilon$

$\varepsilon S \quad \varepsilon S$

Luego $+y \cdot$ tiene únicas extensiones continuas

$+\tilde{\tilde{L}} \times \tilde{\mathrm{L}} \longrightarrow \tilde{\mathrm{L}}$

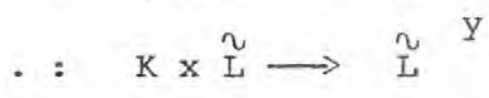

que transforma a $\tilde{L}$ en un e. vect. sobre $k$.

Puesto que $\left\{\mathrm{N}_{\mathrm{V}} / \mathrm{V} \in B\right\}$ es base de la uniformidad $U$ de $\mathrm{L}$, las clausuras $\bar{N}_{\mathrm{v}}$ es base de la uniformidad $\tilde{U}_{\sim}$ de $\tilde{I} \underset{\sim}{\sim}$ además $\bar{N}_{\mathrm{v}}=\bar{N}_{\mathrm{v}_{\sim}} \forall$ veB (Se prueba usando la continuidad de $\tilde{\mathrm{L}} \mathrm{x} \tilde{\mathrm{L}} \rightarrow \tilde{\mathrm{L}}:(\tilde{x}, \tilde{y}) \longrightarrow(\tilde{\mathrm{x}}-\tilde{\mathrm{y}})$.

Luego $\tilde{B}=\{\bar{V} / V \in B\}$ es base de vecindades de 0 en $\tilde{\mathrm{L}}$.

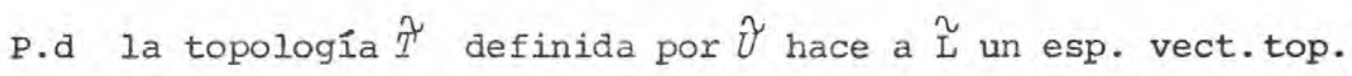

Es claro que $\widetilde{T}$ es invariante bajo traslaciones pues estamos to mando $\operatorname{los} \overline{\mathrm{N}}_{\mathrm{v}}=\mathrm{N}_{\mathrm{v}}$

Además como $B$ satisface:

a) $\forall \mathbf{v} \varepsilon B, \exists \mathrm{U} \varepsilon B$ t.q $\mathrm{u}+\mathrm{u} \mathrm{c} \mathrm{v}$ 
b) $\forall \mathrm{V} \varepsilon B$ es equilibrado y absorbente.

c) $\forall \lambda \in \mathrm{K}, 0<|\lambda|<1$ tal que $\mathrm{V} \in B \Longrightarrow \lambda \mathrm{V} \varepsilon B$ se deduce que $B$ satisface a) y c) puesto que
a) $\bar{U}+\bar{U}=\overline{U+U}$ c $\bar{V} \quad y$
c) $\mathrm{V} \in B \Longrightarrow \lambda \mathrm{V} \in B \Longrightarrow \lambda \overrightarrow{\mathrm{V}} \varepsilon \stackrel{\mathrm{B}}{\Longrightarrow}$

es $\operatorname{decir} \overline{\mathrm{V}} \varepsilon \ddot{B} \Longrightarrow \lambda \overline{\mathrm{V}} \varepsilon \widetilde{B}$

P.d. (b) con lo cual probaríamos que $(\tilde{L}, \tilde{T})$ es esp. vect. top.

Sea $V \in E, \quad \exists U$ vecindad de cero equilibrada t.q

$\mathrm{U}+\mathrm{U}$ c V. Luego $\overline{\mathrm{U}+\mathrm{U}}$ en $\tilde{L}$ es vecindad de cero equilibrada $\mathrm{y}$ $\overline{U+U} \subset \bar{V}$.

P.d que es absorbente.

Sea $\tilde{x} \in \tilde{L}, \exists F$ filtro de Cauchy en $L$ convergente a $\tilde{x}$ y $F \in F$ t.q $F-F \subset v$.

Sea $X_{0} \in F$ arbitrario, puesto que $U$ es absorbente $\exists \lambda C \quad \mathrm{~K}$ t.q $X_{0} \in \lambda \mathrm{U}$ y como $\mathrm{U}$ es absorbente podemos asumir que $|\lambda| \geqslant 1$.

Además $\mathrm{F}-X_{0}$ c U $\Longrightarrow F \subset X_{0}+U \mathrm{y} \tilde{\mathrm{x}} \varepsilon \mathrm{F} \subset \lambda(\overline{U+U})$, es de$\operatorname{cir} \lambda \tilde{x} \varepsilon \overline{U+U} \forall \tilde{x} \varepsilon \tilde{L}$.

La unicidad sale del hecho de que $(\tilde{I}, \widetilde{T})$ como e.v. es único. 


\section{APENDICE.}

Se puede caracterizar un espacio uniforme usando seudométricas o cubrimientos.

1) Vía seudométricas.

Dado $\mathrm{x}$ conjunto no vacío

$\rho: \mathrm{x} \times \mathrm{x} \longrightarrow \mathbb{R}^{+}$es una seudométrica sobre $\mathrm{x}$ si

1. $\rho(x, x)=0 \quad \forall x \in x$

2. $\rho(x, y)=\rho(y, x) \quad \forall x, y \in x$

3. $\rho(x, z) \leqslant \rho(x, y)+\rho(y, z) \forall x, y, z \varepsilon x$

Una familia $\left(\rho_{i}\right)_{i \varepsilon I}$ de seudométricas sobre x está asociada a una uniformidad sobre $\mathrm{x}$ si la familia

$$
S=\{H(i, e) / i \varepsilon I, \varepsilon>0\}
$$

donde $H(i, e)=\left\{(x, y) / \rho_{i}(x, y)<\varepsilon\right\}$ es una subbase de $U$.

\section{Resultado:}

Dada una familia de seudométricas $\left(\rho_{i}\right)_{i \in I}$ sobre $x$, existe una uniformidad $U$ sobre $x$ a la cual está asociada esta familia.

Se puede definir espacio uniforme usando sólo el concepto de seudométrica de la forma siguiente:

Una estructura uniforme sobre $\mathrm{x}$ es una familia $D$ de seudométricas sobre $\mathrm{X}$ que verifica.

1) Si $\rho_{1}, \rho_{2} \in D$ entonces $\max \left\{\rho_{1}, \rho_{2}\right\} \in D$ 
2) Si $\gamma$ es una seudométrica en $\mathrm{x} y$ dado $\varepsilon>0$, existe $\rho \varepsilon D$ y $\delta>0$ tal que

$$
\rho(x, y) \leqslant \delta \Longrightarrow \gamma(x, y) \leqslant \varepsilon
$$

entonces

$$
\gamma \varepsilon D
$$

2) Via cubrimientos.

Sea $\mathrm{X}$ un conjunto no vacío, la familia $C=\left\{A_{\alpha}\right\}_{\alpha} \varepsilon \mathrm{I}$ de subconjuntos de $\mathrm{x}$ es un cubrimiento de $\mathrm{x} \operatorname{si}_{\alpha \varepsilon I} \mathrm{U}_{\alpha}=\mathrm{x}$.

Dados $C_{1}=\left\{_{\alpha}^{A}\right\}_{\alpha \varepsilon I}, C_{2}=\left\{B_{\beta}\right\}_{\beta \varepsilon I}$ dos cubrimientos de $x, C_{2}$ refina $C_{1}$ (ó $C_{2}$ es un refinamiento de $C_{1}$ ) si $\forall B_{\beta}, \exists A_{\alpha}$ t.q $B_{\alpha} \subseteq$ ${ }^{A} \alpha$

Por ejemplo todo subcubrimiento refina el cubrimiento.

Un cubrimiento $B$ de $\mathrm{x}$ es un refinamiento baricéntrico de $C$ si $\{$ st $(x, B) / x \in \mathrm{x}\}$ donde

St $(y, B)=U \quad\left\{B_{\beta} / y \quad \varepsilon \quad B_{\beta}\right\}$

es un refinamiento de $C$.

Definición.

Una familia $G=\left\{C_{\alpha} / \alpha \varepsilon I\right\}$ de cubrimientos de $\mathrm{x}$ es una fami lia uniformizante si

1) Cada par de cubrimientos $C_{\alpha}, C_{\beta}$ en $G$ tiene un refinamiento común $c_{\delta}$ en $\mathrm{G}$. 
2) Cada cubrimiento $C_{\alpha}$ en $\mathrm{G}$ posee un refinamiento baricéntrico $C_{\beta}$ en $\mathrm{G}$.

Resultado.

Sea $G=\left\{C_{\alpha} / \alpha \varepsilon I\right\}$ una familia uniformizante en $\mathrm{X}$, para

cada $\alpha \varepsilon$ I se define

$$
\mathrm{v}_{\alpha}=\mathrm{U}\left\{\mathrm{u} \times \mathrm{U} / \mathrm{u} \varepsilon \mathrm{C}_{\alpha}\right\}
$$

entonces,

$\mathrm{u}(\mathrm{G})=\left\{\mathrm{v}_{\alpha} / \alpha \varepsilon I\right.$ es una uniformidad en $\mathrm{x} . \quad(\mathrm{x}, \mathrm{u}(\mathrm{G}))$ es un esp. uniforme.

Recíprocamente dado $U$ una uniformidad en $\mathrm{x}$, para $\mathrm{V} \varepsilon U$ se define el cubrimiento de $\mathrm{X}$

$$
C(\mathrm{~V})=\{\text { vecindades de } \mathrm{x} / \mathrm{x} \in \mathrm{x}\}
$$

entonces la familia

$\mathrm{C}(U)=\{C(\mathrm{~V}) / \mathrm{V} \varepsilon U\}$

es una familia uniformizante de $\mathrm{x}$.

\section{BIBLIOGRAFIA.}

Bourbaki, N. General Topology. Addison-Wesley. 1966.

Dugundji, J. Topology. Allyn and Bacon. 1966.

Gillman-Jerison. Rings of continuous functions. Springen verlag. 
Kelley, J. Tpología General. Eudeba. 1962.

Schaeffer, H. Topological Vector Spaces. Springer Verlag. 1980. 